\title{
Variations pour flûte sur la fin des Lumières
}

Variations for a flute at the end of the Enlightenment

\section{Michel Vovelle}

\section{(2) OpenEdition}

\section{Journals}

\section{Édition électronique}

URL : https://journals.openedition.org/ahrf/11197

DOI : 10.4000/ahrf.11197

ISSN : 1952-403X

Éditeur :

Armand Colin, Société des études robespierristes

Édition imprimée

Date de publication : 1 septembre 2007

Pagination : $3-28$

ISSN : 0003-4436

\section{Référence électronique}

Michel Vovelle, «Variations pour flûte sur la fin des Lumières ». Annales historiques de la Révolution française [En ligne], 349 | juillet-septembre 2007, mis en ligne le 01 septembre 2010, consulté le 01 juillet 2021. URL : http://journals.openedition.org/ahrf/11197; DOI : https://doi.org/10.4000/ahrf. 11197 


\title{
VARIATIONS POUR FLÛTE SUR LA FIN DES LUMIÈRES
}

Michel VOVELLE

\begin{abstract}
À l'opéra de Moscou, en septembre 2006, une représentation de la Flûte Enchantée où Sarastro portait des moustaches et une casquette a donné à l'auteur l'idée de s'interroger, en remontant d'aujourd'hui à hier, sur l'image des Lumières transposée et transmise par la féerie mozartienne. Visionnant la série des enregistrements de cet opéra depuis un demi-siècle au Festival d'Aix, en élargissant la prospection à d'autres productions singulières - outre le spectacle moscovite, une version cinématographique anglaise en 2007 - il analyse à partir des scénographies et des images le reflet d'une vision et d'idéologies propres à chaque temps. Que sont devenues les Lumières dans l'imaginaire d'aujourd'hui - partagé entre consensus fusionnel et/ou apocalypse totalitaire ? Ce n'est pas l'essai de Régis Debray Aveuglantes Lumières, épinglé in fine, qui nous éclairera sur ce point.
\end{abstract}

Mots-clés : Mozart, opéra, Lumières, Révolution.

« Il me plaît d'entendre un concert de flûtes dans le lointain... ». J'ai toujours aimé citer à mes élèves cette phrase de Diderot dans son dialogue du « Pour " et du « Contre » avec le sculpteur Falconet. Contre la dénégation sans concession de son ami, sur le thème de ce qui reste après la mort, immortalité ou souvenir, Diderot en appelle à la mémoire sous ses différents aspects affectifs. Celle des proches et des êtres aimés, collective ou civique, celle qui se porte sur les individus distingués par leur valeur, leur héroïsme, leur apport aux connaissances mais aussi bien sûr le simple citoyen. Posant les bases d'une nouvelle immortalité, affranchie de toute référence métaphysique ou religieuse, il esquisse les voies qui conduiront au culte des morts tel que le XIX ${ }^{\mathrm{c}}$ siècle le banalisera. 
Voilà bien un des héritages, non des moindres, dont nous sommes redevables aux Lumières, et qui a transité par la Révolution française, expérience gigantesque au sein de laquelle les nouvelles valeurs, les nouveaux rêves énoncés par les Lumières ont été mis à l'épreuve.

C'est pourquoi je me suis senti sollicité, à mon niveau de tâcheron de l'histoire des Lumières et de la Révolution, par l'étrangeté de la séquence que nous vivons au regard de cet héritage. Dans le contexte plus général de l'esprit du jour, deux mises en question m'ont interpellé particulièrement : la lecture de l'essai de Régis Debray Aveuglantes Lumières', s'enchaînant sur une expérience plus particulière, une soirée d'opéra en septembre 2006, à Moscou, où l'on donnait la Flûte Enchantée dans une présentation originale. De fil en aiguille, je me suis trouvé à reformuler la question naguère posée par J.M. Goulemot : «Que reste-t-il des Lumières? »", en me laissant porter aux accents des avatars et tribulations de l'opéra de Mozart, comme de la façon dont il a été compris et reçu dans les dernières décennies.

Est-il besoin de rappeler aux lecteurs des $A H R F$ l'argument de la Flûte ? Dans un monde enchanté s'affrontent les puissances obscures de la Reine de la Nuit et celles du Sage Sarastro, entouré de ses adeptes. À l'issue d'un parcours semé d'épreuves, le jeune prince Tamino accède à la vraie sagesse dévoilée aux initiés, en même temps qu'à l'amour de son élue Pamina. La Flûte est une fantaisie, un Singspiel dont les aspects féeriques tragicomiques s'enrichissent de la présence du couple populaire, Papageno l'oiseleur et sa compagne Papagena. Mais de façon à peine voilée la transposition par Mozart et son librettiste d'une initiation de type maçonnique donne à cette aventure la hauteur et la gravité d'un message de foi dans l'avènement des Lumières. Espièglerie pour les uns, mystère pour les autres, depuis deux siècles l'enchantement a perduré.

\section{Aix - Cinquante ans de festival}

En près de cinquante ans, de 1958 à 2006, le festival d'Aix-enProvence a inscrit quinze fois la Flûte Enchantée à son programme. Je n'ai pas assisté à toutes les représentations, on s'en doute - quatre à cinq si je me souviens bien, de la création (locale) à hier. Mais piqué au jeu de cette recherche de mémoire, j'ai visionné à la médiathèque d'Art Lyrique d'Aix ${ }^{3}$ une douzaine de coffrets vidéo, supports précieux, précaires aussi. Suffisants toutefois en élargissant la recherche (sans ambition d'exhaustivité)

(1) Régis Debray, Aveuglantes Lumières, Paris, Gailimard, 2006, 204 p.

(2) Jean-Marie Goulemor, Adieu les philosophes, que reste-t-il des Lumières ?, Paris, Seuil, 2001.

(3) Médiathèque d'Art Lyrique - Cité đu Livre. Bibliothèque Méjanes, 8-10 rue des Allumettes, 13090 Aix-en-Provence. 
pour suivre un fil continu, celui de la façon dont le Singspiel mozartien a été présenté, « scénario », décors et images, esprit, entre fidélité à un héritage et souci de modernisation, allant jusqu'à une relecture complète. Pour ce faire, j’ai complété la leçon des images fixes ou mobiles par la lecture des livrets, interviews, et comptes rendus que j'ai pu rassembler. Et bien sûr, persuadé qu'Aix n'est pas le centre du monde (même si une partie de ces « créations » ont tourné de Salzbourg à Paris ou autres lieux), j'ai comparé à des enregistrements en DVD (Natalic Dessay au Palais Garnier par exemple), comme aux films, ma référence initiale étant, comme pour beaucoup d'autres, le chef-d'œeuvre d'Ingmar Bergman, image forte dans sa dignité et sa sobriété... pour aller jusqu'à la provocation récente à laquelle je réagis ici in fine, la production du réalisateur anglais $\mathrm{K}$. Branagh, une surprise de Noël 2006.

On pourra craindre que le regard privilégié que j'ai de longue date porté sur l'image, sous toutes ses formes, ne m'entraîne à survaloriser celles-ci : mais je crois qu'elles offrent la possibilité de suivre sur un demisiècle les métamorphoses du regard porté sur l'illustration mozartienne du message des Lumières. Ce que je fais, non pas en expert, mais non plus en dilettante, en historien des idées et des représentations, vocation qui ne date pas d'hier'.

Dans ma jeunesse, j'avais été passionné par les biographies de Jean et Brigitte Massin dans les années cinquante ${ }^{f}$, et j'avais aussi comme tout un chacun mon livret commentaire de référence - l'ouvrage de Jacques Chailley sur "La flûte enchantée opéra maçonnique »". Je me suis même risqué, profane en tous genres, à évoquer la Flûte Enchantée à partir de la belle version filmée qu'en donna Ingmar Bergman, dans un article de revue (Temps Libre, 1982), en l'associant à deux autres productions cinématographiques - L'oiseau bleu et L'âge de cristal - sur le thème "Pour une nouvelle histoire du rêve ", qui fut repris dans mon ouvrage Histoires figurales en 1989. Cet essai - esquisse sur une piste que je n'ai pas approfondie à défaut de me qualifier pour disserter en expert sur la Flûte Enchantée justifie peut-être que j'y revienne, un quart de siècle plus tard, pour illustrer à partir d'une étude de cas emblématique, ce que je dénommerai les travestissements de la mémoire (ou de l'héritage) en l'occurrence, à propos des Lumières.

Que la présentation scénique - décors, costumes, artifices et traitement des épisodes - ait considérablement changé ne saurait étonner.

(4) Consultés à la Médiathèque, et pour certains aux bureaux du Festival.

(5) La Révolution française images et récit, Paris, Messidor, 1986 ; Histoires figurales, Paris, Usher, 1989 ; Les âmes du Purgatoire ou le travail du deuil, Paris, Gallimard, 1996.

(6) Jean et Brigitte Massin, Wolfgang Amadeus Mozart, Paris, Le Club français du livre, 1959.

(7) Jacques CHall.LEY, Musique et ésotérisme. La Flate Enchantée, opéra maçonnique, essai d'explication du livret et de la musique, Paris, R. Laffont, 1968. 
Il était inévitable que l'on prenne des libertés, même si le scénario, dans ses composantes fondamentales impose des contraintes ; une évolution s'est imposée, qui n'est pas spécifique de l'opéra de Mozart mais surtout, la nature même de l'argument de cette fantaisie sérieuse risque de n'être plus perçue d'une grande partie du public.

Qui a lu Jacques Chailley aujourd'hui ? Je n'ai pas la vanité de croire que je suis le seul - moi et ma génération - ; et les livrets présentés souvent avec finesse et pédagogie (Jacques Amblard - 2001 - Tzvetan Todorov et Krystian Lupa - 2005-2006) ${ }^{k}$ rappellent la trame d'une initiation maçonnique sur plusieurs registres, en l'enrichissant de problématiques d'aujourd'hui (le « genre» ou la guerre des sexes), voire de l'épanchement de leur sensibilité personnelle (Michel Del Castillo, «Les opéras du festival d'Aixen-Provence »-1982)". Mais, même annoncé, le discours initiatique crypté, porté non sans ambiguïté par les tribulations des héros risque bien de ne plus passer aujourd'hui, devenant une fantaisie dont on ne comprend pas la langue, pour se livrer au charme intemporel de la musique... et éventuellement des images.

Voilà qui laisse au metteur en scène et au décorateur qu'il inspire une bien grande liberté, mais aussi une responsabilité considérable. Celle d'exprimer dans notre langue d'aujourd'hui un message qui se révèle, plus encore que dépassé, susceptible de travestissements multiples. L'histoire contemporaine retrouve ici sa place, aspirant à redonner un sens ou un souffle à un héritage fatigué, l'aggiornamento peut se faire suivant plusieurs démarches, par transposition directe ou indirecte. Le " tragique " $\mathrm{XX}^{c}$ siècle fournit des références tant pour la mise en scène et l'anachronisme voulu, que plus profondément sur le sens profond de l'œuvre. Ainsi n'ai-je pas été surpris, pour ne prendre qu'un exemple, de voir Fidelio à l'Opéra Bastille inséré dans le cadre d'un univers concentrationnaire, camp ou goulag.

Mais, pour en rester à la Flûte Enchantée, la retranscription, telle qu'elle se voit pratiquée depuis au moins une vingtaine d'années, ne peut se faire sans s'attaquer à la signification profonde du sujet et au message dont il est porteur. Au-delà de l'objectif énoncé d'actualiser l'œuvre, en la débarrassant de son ésotérisme maçonnique suranné, ce qui est bien en question, c'est la signification et la pertinence du discours, comme de l'idéal des Lumières, tel qu'il a été transmis par la Révolution française.

Aix avait sa tradition, en deçà de laquelle je m'abstiendrai de remonter en sollicitant d'autres héritages, sur d'autres scènes : cela nous entraînerait

(8) Wolfgang Amadeus MozART, Die Zauberflöte, Festival International d'Art Lyrique d'Aix-enProvence, Livret 2001, Jacques AMBLARD, "La composition de la Flûte », p. 24-29; Livret 2006, Tzvetan TODOROV, « Entre lumière divine et lumières humaines ", p. 12-13; Krystian LuPA, "Introduction \#, p. 6-10.

(9) Collectif, Les Opéras du Festival d Aix-en-Provence, Aix, Edisud, 1982. 
à un flash back passionnant sans doute mais disproportionné. Au demeurant, c'est un certain " classicisme " qu'évoque pour moi la présentation entre 1959 et 1965 sous des baguettes différentes, mais la mise en scène constante de J.-P. Grenier dans les décors de J.-D. Malclès, avec le souvenir particulièrement vif de celle de 1963 dirigée par John Pritchard - Gundula Janovitz tenant le rôle de Pamina. Aix était véritablement abonné à la Flûte : cinq représentations en sept ans! Mais l'esprit restait le même, tel que le présentateur l'évoquait en 1963 : un dialogue pathétique entre " obscurité et lumière ", « haine et amour " ${ }^{10}$. Un prince sauvé par des fées se réveille-t-il ou poursuit-il son rêve ? Mais le conte, ou la féerie devient autre chose : une fable, incarnant l'esprit de la franc-maçonnerie, d'où le danger d'une ambiguitté transcendée par le génie de Mozart. Un Mozart qui l'assume, plus proche, dans ce combat entre le bien et le mal, de Papageno sans doute, humain, trop humain, mais attentif dans l'affrontement entre ce que nous sommes et ce que nous voudrions être à faire prévaloir la sagesse, c'est-à-dire l'esprit.

On peut aujourd'hui trouver datés les décors - les rochers de carton pâte de la "forêt sauvage ", les architectures égypto-assyriennes du second acte, de l'initiation à l'apothéose finale présidée de son trône par Sarastro sur fond de soleil resplendissant. Tamino apparaissait comme une sorte de jeune guerrier plutôt à la romaine, la Reine de la Nuit surgissait en robe décolletée noire relevée d'un large collier et surmontée d'un haut diadème, cependant que sa fille en robe panier blanche se contentait d'une petite couronne plus modeste. Les trois dames de la Reine en noir apparaissaient voilées, et dans la scène initiale porteuses de lances, puis de torches lors de leur seconde visite. Couronnés également les trois enfants évoquaient plutôt le style pages florentins, ou lutins comme on voudra, la fantaisie était incarnée par Papageno en termes de plumes multicolores reprise d'un modèle connu dans l'iconographie du personnage mis au goût du jour. Sarastro et ses fidèles donnaient délibérément dans le style assyrien : barbes, longues robes, turbans (et couronnes!), le mage se distinguant par un soleil brodé comme par la hauteur de sa coiffe. Tout ceci restait assez hiératique et somme toute très digne, et nous sommes restés marqués par cette référence.

Lorsque je renoue le fil des images, en 1989 plus de quinze ans ont passé, dont onze sans Flûte, avec des moments difficiles pour le festival. Armin Jordan dirige l'ensemble orchestral de Paris, Jorge Lavelli signe la mise en scène, Helen Kwon, ravissante Reine de la Nuit, meurt de peur de manquer ses trilles. La nouveauté ne va pas jusqu'à l'impertinence outrée. Le paysage initial surprend : une rocaille porteuse d'une forêt de sapins

(10) Enregistrement vidćo de la première représentation, Médiathèque d'Art Lyrique d'Aix-en- 
nordiques, ce n'est pas à cet exotisme que nous sommes accoutumés. Tamino est vêtu d'une tenue blanche légère, avec une grande écharpe, la surprise vient du serpent, un grand lézard multicolore, qui, son numéro exécuté, va quitter sa dépouille pour saluer sous les traits d'une danseuse en tutu blanc. Les trois dames en noir, la tête complètement couverte d'un voile qui cache leur chevelure blonde (qu'on retrouvera chez la Reine en robe noire à paillettes), montent un intermède assez comique, dansant avec les balais qui les identifient comme des sorcières. Papageno a perdu ses plumes, en culottes courtes et tenue rustique, il traîne une petite cage à roulettes abritant ses oiseaux. C'est là une des fantaisies que désormais on s'autorise et que l'on relève quand elles se rencontrent par la suite : ainsi de faire venir à bicyclette les trois enfants en costume marin, ou introduire Pamina, classiquement vêtue de blanc, sur une chaise longue roulante façon 1900, dans la périlleuse scène du deuxième acte où elle est exposée aux assauts de Monostatos. Malgré quelques touches de nouveauté - les deux hommes en armes aux portes du sanctuaire, hautes silhouettes à jupe rouge - la tonalité d'ensemble reste d'une sobriété classique, le prêtre (ou l'orateur) qui accueille Tamino est vêtu d'une robe de bure blanche, comme le chœur d'allure très monacale. Sarastro se distingue par la hauteur de son turban, dans un décor à l'égyptienne en trompe-l'cil, où les évolutions se déploient respectueusement dans une ambiance de péplum encore statique.

Il semble bien qu'en ces années on restait partagé entre respect et irrespect, désir de prendre ses distances avec la littéralité du message, et crainte de faire apparaître - qui sait - la gratuité ou la vacuité d'une « fable » à laquelle on avait peine à se rattacher. Cette ambiguïté, d'ailleurs, n'était pas propre à Aix-en-Provence : de Paris, au Palais Garnier nous viennent les images d'une présentation assez récente, qui mit en valeur Natalie Dessay, belle Reine de la Nuit en robe bleue, micux servie que ses suivantes, et dont je retiens surtout au milieu d'intermèdes assez plaisants (le ballet des animaux envoûtés par la musique au second acte) le parti-pris d'avoir revêtu Sarastro et les siens de grandes chapes richement décorées de type copte peut-être, ou intentionnellement maçonniques, par les crachats qu'ils portent, témoins d'un rite imaginaire. Sarastro, au crâne hypertrophié impose sa présence. Le clin d'œil vient de ce qu'il laisse entrevoir, sous sa robe le col d'une veste encadrant une chemise cravate. Est-ce le bon moyen de moderniser la Flûte ? Reste la solution de tout remettre en cause. 


\section{4 : un nouveau regard}

En 1994, retrouvant après une absence le chemin du Festival d'Aix, j'ai pu profiter comme beaucoup d'autres, de ce qui a été salué comme une mise à neuf de la Flûte Enchantée, sous la direction de William Christie, dans la mise en scène du québécois Carsen, les décors de Kilmontk représentant une tentative inégalement heureuse à mon goût de s'adapter aux contraintes de l'espace scénique du théâtre de l'Archevêché - ainsi dans l'utilisation des loges latérales pour certaines séquences acrobatiques (mais accrocher en l'air à des pilastres les enfants comme des pantins était assez gratuit). Ce ne serait que chicane, et l'on pouvait accepter le parti d'une scène dépouillée, sombre et monochrome en rouge dans les scènes "chaudes" (comme celle du début) et plus souvent en bleu, voire dans une obscurité éclairée par en bas d'une lumière vive. Ce sont là artifices qui ne mettent pas directement en cause le déroulement ni la vision d'ensemble. Le choix des costumes donne une indication plus directe de l'esprit dans lequel s'inscrit le metteur en scène. Un Papageno en tenue rustique de chasseur des bois, égayée chez sa compagne d'une coiffe en forme de nid d'oiseaux n'avait rien de provocateur. Que Tamino découvert inanimé en tee-shirt, et caleçon blanc se voie revêtu d'une sorte d'uniforme galonné de diplomate ou de préfet - pourquoi pas ? Pamina comme sa mère la Reine de la Nuit toutes deux en robe de soirée bleue Second Empire : voilà deux divas presque jumelles - la mère se porte jeune. Avec ce parti-pris d'une chronologie modérément aggiornamentée, on ne s'étonne pas que, lorsque Sarastro déboutonne sa soutane au deuxième acte, il apparaisse en uniforme blanc d'officier de marine américain. Il reste qu'il porte cette sorte de soutane blanche, comme les «prêtres » et assistants qui l'entourent et forment un chœur, voilé dans une partie des scènes, sans être tout à fait des pénitents encagoulés, plutôt parfois une procession de fantômes. Peut-on parler de " cléricalisation » de l'opéra maçonnique ? À l'entracte, dans la présentation enregistrée sur Antenne 2, la commentatrice Ève Ruggieri taquine le metteur en scène d'Outre-Atlantique qui se défend en invoquant l'esprit du lieu, cet ancien Archevêché qui invite à s'adapter à une "culture catholique ", mais avec liberté, de façon moderne. Telle qu'il l'entend, la modernité provocatrice est d'avoir déguisé les trois suivantes de la Reine de la Nuit en nonnes en robe rouge écarlate, tenue adaptée à leurs écarts plaisants, voire provocateurs : car si sur la cassette officielle on n'en a donné qu'unc version honnête, je ne suis point seul, ni victime d'un fantasme à avoir constaté au fil de leurs galipettes que leurs porte-jarretelles étaient dans la même tonalité. 
Va pour les nonnes en folie, comme provocation initiale, mise en appétit (?) du spectateur prévenu qu'il ne faut pas prendre les choses trop au sérieux. Mais la scénographie comporte un certain nombre d'innovations qui prennent résolument leurs distances avec le cadre pseudo égyptien du livret traditionnel. On peut classer au rang des trouvailles la présence récurrente - dans les premières scènes - puis au second acte dans celle de l'initiation, d'un arbre, une sorte de baobab, volumineux, présence de la nature, propre à éclairages divers, transformable en coffre, ou buffet dont on tirera les accessoires de l'initiation comme il délivre la flûte magique. Larbre en feu se substituera aux deux montagnes, embrasées ou ruisselantes du livret original, si difficiles à matérialiser. On ne s'en offusquera pas, tout en notant que le cérémonial de l'initiation s'en trouve pour le moins modifié dans son esprit comme dans ses gestes. Mais on franchit le scuil des simples licences, lorsque, au début de l'acte deux, la procession des pénitents blancs voilés prend place autour d'une très longue table, présidée par Sarastro pour délibérer sur le sort à réserver aux candidats à l'initiation : "pénitents » et/ou prêtres... porteurs de calices enflammés ; on reconnaît dans cette assemblée où la mixité est de rigueur les trois enfants, les dames de la Reine de la Nuit, les officiants, rassemblés dans un cadre qui tiendrait plus de la quête du Graal que d'une tenue maçonnique. Mais la surprise est grande de voir la table se transformer en plateau scénique lorsqu'y pénètrent, voilés et titubants, Tamino et Papageno dont la rencontre en forme de pitrerie (certes comme dans le livret mais accentuée) achève de désacraliser la scène. Elle retrouvera quelque mystère par la suite, lorsque le metteur en scène imagine de faire porter processionnellement à ses prêtres-choristes une forêt d'arbres morts assez shakespearienne pour s'acheminer vers le lieu de l'épreuve. Mais il nous aura fallu au préalable affronter une autre surprise. Elle est préparée par le numéro de Monostatos, à qui incombe la charge peu gratifiante du harcèlement sexuel sur la personne de Pamina, endormie, désespérée. Comment assumer aujourd'hui un rôle politiquement si déshonnête, et qui dévoile un Mozart bassement raciste, dans sa dénonciation des races inférieures? Christie et Carsen, aidés par la présence de leur interprète Cole, habitué du rôle, un "black" bien sympathique, en font un partenaire présentable, dont on verra la consécration au final de la pièce, lorsque Sarastro lui donnera une accolade fraternelle.

Il ne suffit pas, à l'heure du rejet des discriminations de cette petite prouesse : au cœur de l'intrigue, on le sait, le thème lancinant de la guerre des sexes constitue un ressort essentiel, auquel sans doute nous sommes plus sensibles en un temps où le problème du « genre " (traduction littérale du gender anglo-saxon) peut nous faire regarder avec une sévérité 
incompréhensive le machisme mozartien. La femme, être inférieur, soumise à ses passions, agent de la superstition, force du mal en la personne de la Reine de la Nuit : cette vision traditionnelle qui réserve à l'homme l'accès aux Lumic̀res, élargi par privilège à sa compagne ne rendelle pas obsolète le message mozartien ? Notre équipe anglo-saxonne a résolu le problème. Une fringante Reine de la Nuit en la personne de Nathalie Dessay, qui tient à préciser dans une interview qu'elle n'entend être ni mégère ni harpie, se présente comme une très jeune mère. Et finalement, dans la scène où Sarastro se justifie auprès de sa fille éplorée de ce que l'on appellerait aujourd'hui l'échec de son mariage, on voit s'asseoir, à l'autre bout du lit, inattendue, silencieuse et émue... la Reine de la Nuit. Ils sont réconciliés! Mozart n'avait pas prévu cela, et plusieurs spectateurs sont surpris. Reste qu'il pouvait, sur ces bases, paraître difficile de mettre en scène l'intrusion suivante de la bande des conspirateurs du souterrain : Monostatos, la Reine et ses suivantes... Expédié vite fait bien fait, cet escamotage s'achève comme il se doit sur un coup de tonnerre mais qui n'a rien de cataclysmique, puisqu'il introduit un final triomphal. C'est le grand pardon : tous en blanc, les acteurs grands et petits ont droit chacun à une couronne, Monostatos ému reçoit la bise de Sarastro, dans un cérémonial plus postconciliaire que maçonnique, on se donne la paix en se serrant la main comme à l'église avant de former un grand cercle d'amitié... après quoi dans une sorte de striptease collectif, tous se débarrassent de leur lourde soutane blanche, pour apparaître en sous-vêtements forts décents (plus question de porte-jarretelles). Le triomphe des Lumières sur les forces du mal se conclut par « Tout le monde il est beau, tout le monde il est gentil ».

Je crains qu'on ne me soupçonne d'avoir gardé rancune à l'égard de ce spectacle qui n'était pas sans invention ni sans mérite, à défaut de transmettre le message de Mozart... et des Lumières tel qu'on l'entend. Peutêtre ai-je été agacé de la contagiosité du consensus tel qu'il s'est exprimé dans la presse, quasi unanime sur le dépoussiérage heureux d'un chefd'œuvre vieilli : Le Monde, comme L'Humanité ont chacun dans son style salué cet effort de modernité, sans prendre la mesure semble-t-il des dégâts. William Christie était fier de son « espièglerie », Carsen se félicitait d'avoir gommé l'aspect " bataille de sioux contre cow-boys » qui l'agaçait pour célébrer une "leçon de vie » accessible aux petits comme aux grands. Et Ėve Ruggieri y voyait une leçon de « fraternité, de générosité et de tolérance $»$ (ainsi soit-il)".

La version aixoise de 1994-95, déconstruction du message mozartien ne s'est pas imposée comme une nouvelle vulgate, et sans entreprendre de

(11) Interviews transcrites d'après l'enregistrement de la première séance. 
suivre les étapes durant les dix dernières années, des représentations non seulement aixoises $(1999,2001)$ mais internationales (Aix - Vienne Luxembourg 2006) nous pouvons évoquer quelques repères. L'" aggiornamento formel » suit son cours, dira-t-on, dans un raccourci certes caricatural : Tamino en pyjama entouré de sbires en robe ou costume de bure à ceinture de corde en 2001, sur un fond de décor assez carcéral, personnages vêtus ou dévêtus jusqu'à la nudité extatique de Tamino et Pamina " Heïlige Menschenpaar " en 2006'.

Ces versions reflètent les caprices et difficultés d'adapter la féerie à la postmodernité. Mais l'esprit de l'œuvre n'était pas l'objet d'une relecture frontale, lorsque Jean Amblard en 2000-2001 commentait, dans une veine éprouvée le mariage du texte et de la musique, un hymne à l'amour humain transfiguré par les prestiges du chant ${ }^{13}$. Le plus récent livret (2005-2006) $)^{14}$ de la coproduction (Aix-Vienne) dirigée de l'orchestre par Daniel Harding et le Mahler Chamber Orchestra, dans la mise en scène de Christian Lupa ouvre, sous une direction germano-polonaise, sur un certain esprit du temps, quinze ans après la chute du mur de Berlin. Mozart - et sa vision des Lumières - n'y sont pas agressés, mais n'en sortent pas indemnes. Dans une intervicw avec Anna Lebedezka, le polonais Christian Lupa redistribue les rôles, accentuant une tendance déjà perceptible ${ }^{15}$ : pour lui le véritable héros est bien Papageno, humain, proche de la nature comme Kaspar Hauser, l'enfant sauvage, mais conquérant son humanité, à travers l'affirmation érotique, et surtout par le pouvoir de dire non, de résister à toute forme d'« initiation " aliénante. Papageno pour le commentateur polonais est le "maître à penser des insoumis ». Le personnage de Tamino n'en ressort pas grandi. Comment expliquer son coup de foudre sinon par l'influence quasi hypnotique de la femme, en l'occurrence de la Reine de la Nuit ? Tamino sera un faux initié car pas plus que Papageno il n'assume ni ne refuse le cheminement qui lui est imposé.

La réflexion de Christian Lupa est élargie par Tzvetan Todorov, qui ne se gêne pas pour dire ce qu'on n'osait formuler ${ }^{16}$ : Sarastro censé incarner sagesse et perfection a des esclaves qu'il maltraite, il règne en despote sur ses sujets, convaincu de détenir la raison, prônant le pardon, il pratique la vengeance, dans un univers manichéen où s'opposent avec la nuit et le jour, le bien et le mal, la passion et la sagesse. L« axe du mal », comme l'écrit l'auteur, s'incarne dans les figures emblématiques de la femme et du noir, être inférieurs et nuisibles.

(12) Wolfgang Amadeus MozART, Die Zauberflöte, livret, op. cit. note 8, p. 11, 12, 15.

(13) Jean AMBARD, La composition de la Flûte, op. cit. note 8.

(14) Op. cit. supra note 12 .

(15) Op. cii. supra note 8, Krystian LuPA, \& Introduction ", p. 6.

(16) Op. cit. supra note 8 , Tzvetan ToDorov, * Entre lumières divines et lumières humaines ", p. 12. 
Que reste-t-il de l'hymne à l'amour humain, celui même qui nous émeut jusqu'au tréfonds quand les voix de Tamino et Pamina se mêlent dans le franchissement des épreuves ? C'est le prestige de la musique que de nous le faire percevoir. Mais pour Sarastro qui l'exploite, ce n'est pas l'amour qui prime. Et Todorov, après Lupa, insiste sur la dualité de deux voies d'accomplissement, l'une sacrée qui passe par l'initiation et les mystères, l'autre toute humaine : c'est celle des Lumières humanistes, celle de Papageno faussement interprété comme préférant la matière à l'esprit. Et c'est bien celle que préfère notre auteur, en rappelant l'ambiguité de Mozart lui-même dans son appréciation du personnage, avec qui il s'identifiait. Dans cette relecture des deux voies d'accès aux Lumières, malgré la distance prise avec Sarastro, porte-parole officiel, les Lumières ne sont pas contestées ; mais il y a plusieurs filières pour y accéder.

\section{Moscou 2006. Les moustaches de Sarastro}

À la fin de septembre 2006, je suis repassé à Moscou que je n'avais pas revue depuis le printemps 1989. Faut-il dire que je l'ai trouvé changée? Une surprise me fut réservée à la fin du séjour, quand ma collègue Madame Namazova récompensa l'assiduité des participants français par une soirée à l'opéra, pour une première représentation de la Flûte Enchantée dans une nouvelle mise en scène ${ }^{17}$.

À Moscou, une autre étape a été franchie. La version de la Flûte que j'ai eu le privilège de voir tient une place singulière dans la série des métamorphoses de l'opéra mozartien, même si, a posteriori, clle apparaît très significative en ces temps et en ces lieux. Là où l'on pouvait gratifier la version aixoise de Carsen en 1994-95 d'une certaine dose d'innocence dans sa fantaisie innovatrice, la relecture qui nous est proposée ici est délivrée sans détours dans la mise en cause explicite du message des Lumières, à l'épreuve de l'expérience totalitaire du $X^{c}$ siècle. Qu'on en juge à partir de la mise en scène d'un réalisateur allemand, mais à l'intention évidente d'un public russe, qui se veut provocatrice tant sur le fond que dans la forme.

On peut présenter comme une contrainte constante du livret la symbolique des trois portes, qui enferment le lieu d'une initiation : elles délimitent d'entrée un lieu scénique dépouillé, fond de tableau d'un rivage sur lequel s'échoue Tamino, un touriste ahuri, débarquant en costume marin avec son appareil photo dans une forêt que l'on pourrait supposer amazonienne, où il va subir d'entrée les brimades espiègles de naturels (ou de singes ?) qui le lui chipent, - ce sont en fait les trois enfants que nous

(17) Le 30 septembre. 
allons retrouver, dans leur style canaille au fil du livret. Comme on peut s'y attendre ce n'est pas là le pire : un serpent gigantesque - qui se déplie depuis la salle - l'agresse de sa tête assez obscène. Effets faciles non évités mais recherchés, que suit l'apparition des dames de la Reine de la Nuit. Mais leur entrée attendue permet de prendre conscience d'une autre dimension de ce lieu clos, que l'on découvre sous surveillance dans ses combles : de sa rambarde guette la Reine de la Nuit, observant la scène et dirigeant les opérations. Les jeux sont faits... que représente-t-elle pour le réalisateur ? Il l'a soignée, mais pas flattée, il est vrai que je l'ai trouvée maigre dans sa robe d'un rouge violent, ouverte jusqu'au nombril, dévoilant à demi des seins que découvrent plus qu'elles ne cachent deux pastilles noires en forme d'étoile. Un maquillage agressif, la coiffure surmontée du croissant de lune qui la désigne, la Reine de la Nuit a les mains prolongées par des ongles vernis d'une longueur démesurée. Détail qui se retrouve chez ses trois suivantes, belles filles aux cheveux tarabiscotés, chacune à sa manière, et pour le reste vêtues d'une robe noire à étoiles blanches, identique à une nuance près qui ne passe point inaperçue : l'insertion dans cet ensemble d'une découpe blanche moulante très près du corps qui chez l'une d'elles «colle » au torse jusqu'au pubis, et dévoile chez une seconde non seulement l'échine mais une paire de fesses provocante. Ce ne serait là encore que hors-d'œuvre auxquels nous sommes désormais accoutumés, nous en avons vu d'autres avec les porte-jarretelles des nonnes lubriques du réalisateur québécois, et nous ne nous étonnons pas de l'ardeur sans complexes avec laquelle elles raniment Tamino. Par référence, l'apparition de Papageno, vêtu de vert comme il se doit, mais sans trop de fantaisie remet, si l'on peut dire sur le droit chemin avec ses gadgets, et ses épisodes : mais la voix et la présence en scène de l'interprète aidant, nous avons conscience de trouver à côté d'un Tamino bien pâlichon (mais n'est-ce pas aussi dans son rôle de « ravi »?) un protagoniste majeur : quel sera le vrai héros de l'histoire?

La vraie surprise est à venir : nous ne tarderons pas. Mais nous aurons au préalable fait la connaissance de Pamina, belle voix pour un personnage que le décorateur a vêtu, sans trop d'apprêt dirait-on, d'une robe courte de confection décorée d'étoiles et qui s'applique à recoller le croissant de lune qu'on lui a infligé sur le front : c'est Monostatos qui a été particulièrement soigné, transformé en une sorte de Bibendum blanc et boursouflé ; un moyen peut-être de suggérer sa bestialité en éludant le problème de la négritude.

Les séquences d'approche, et de distribution des rôles, avec le temps fort du grand air de la Reine de la Nuit, en sorcière redoutable (que représente-t-elle : la nature, la femme, la face de l'ombre ?), débouchent sur ce 
qui sera la surprise majeure d'un parcours que nous ne suivons pas dans le détail, car il est - du moins jusqu'au final - globalement respecté, mais qui nous découvre avec le palais de Sarastro, l'utopie réalisée.

Livrons-en tout de suite le thème : un officiant en uniforme, avec casquette et moustaches, astique avec zèle la statue d'une blancheur immaculée d'un autre moustachu assis, en qui nous reconnaissons sans peine un personnage historique connu. Eh quoi ! Sarastro serait-il Staline ? Nous n'en doutons plus à la scène 18 quand il fait son apparition en personne, un peu plus grand que nature semble-t-il (une illusion peut-être ?), sanglé dans son uniforme, souriant sous ses moustaches, l'expression inquiétante du «prince bienveillant "suivant un terme repris d'Alain Besançon mais qui saura révéler sa vraie nature lorsque sa fille Pamina résistera à ses exhortations, lui tordant le bras en père brutal avec qui on ne plaisante pas. Son palais est une forteresse, prenant à l'occasion l'allure d'un camp lorsque sa muraille se hérisse des gardes armés, ses plus proches acolytes semblent clonés sur lui, sanglés dans des uniformes, voire statufiés vivants comme les deux " hommes cuirassés " traditionnellement malaisés à faire passer sur la scène. Comme il a ses sbires il traîne derrière lui cet esclave, Monostatos dont nous avons vu la difficile incarnation grotesque.

Aussi délicat était de représenter le groupe des élus, entendons des initiés, qui jouent un rôle important dans le dispositif scénique puisqu'il leur revient de constituer les chœurs. À ce stade, le metteur en scène, visiblement a hésité, transformant leur première intervention en un défilé qui serpente du devant au fond de la scène : masques identiquement figés de moustachus à casquette (en version russe de contrôleur de trains) porteurs sur l'épaule d'un petit marteau... Nous les retrouvons sous différentes variantes : en chasseurs armés de fusils, en garde armée, voire dans un des épisodes, travailleurs actifs, qui s'affairent mollement à quelque chantier stakhanoviste dérisoire. La discipline règne, à peine infléchie par un mouvement de curiosité collective quand les deux héros, Tamino et Pamina entrent dans la fournaise de leur épreuve initiatique suggérée derrière la cloison. Le haut de la scène se transforme en panopticon, à cela près que deux postes d'observation rivalisent : celui de Sarastro et celui de la Reine de la Nuit. Mais qu'advient-il des deux personnages introduits dans cet univers totalitaire ? Tamino, le petit marin, reste égal à lui-même, docile sinon à un lavage de cerveau, du moins à l'épreuve du silence et de la communication interdite. On dira que le secret maçonnique se prêtait à cette transposition. Pamina plus vibrante résiste mais à dire vrai cette version, pas plus que beaucoup d'autres, ne nous livre le secret du revirement intérieur des deux protagonistes. C'est Papageno qui l'emporte, comme souvent, à la fois par le talent de l'interprète et par les facilités d'un 
rôle qui fait de lui non seulement le héros proche, mais celui qui obstinément dit non à la normalisation; il ne sera pas un élu à part entière, mais on lui concède les joies de la famille et le metteur en scène en rajoute en glosant sur le couplet de Mozart qui promet un tas de petits Papageno et Papagena, il en fait surgir cinq qui gambadent sur scène dans le final.

Celui-ci pourrait donc, comme dans la version québécoise être le prélude à un happy end consensuel, et d'une certaine façon c'est bien ce qui advient, car nous verrons finalement Tamino reprendre son voyage non seulement avec Pamina mais avec la tribu des Papageno dans le petit bateau (élargi) sur lequel il est arrivé. Mais la vraie fin est déjà advenue : sorti de ses souterrains, le commando des terroristes, Monostatos, la Reine de la Nuit et ses trois suivantes a fait irruption sur la scène, et pour que nul n'en ignore, l'une des suivantes porte un missile (en réduction) et l'autre un avion ; leur explosion laisse apparaître un champ de ruines et de cadavres de la garde de Sarastro, dont on entrevoit la tête pendante à sa lucarne... sort partagé par la Reine de la Nuit, à la sienne.

Ainsi la Terreur refait-elle son apparition sous sa forme la plus brutale, animée par les forces du " parti du mal », associées à la bestialité populaire (Monostatos). Le totalitarisme « soft », dont Sarastro était l'incarnation sous des dehors occasionnellement débonnaires, s'écroule dans une apocalypse finale dont les protagonistes ressortent vaincus et anéantis. Et nous qui attendions de communier dans l'apothéose des Lumières... "die Strahlen der Sonne vertreiben die Nacht... ", nous voici fort déconfits de constater qu'il n'existe qu'une voie de salut : "Sauve qui peut »... la formule est de Voltaire, mais ce n'est pas celle que nous avions attendue du message mozartien.

À chaque époque la mémoire se colore et s'entretient de ses rêves collectifs, ou au contraire de ses déceptions voire de ses désespérances. Elle règle ses comptes avec le passé, avec les "illusions " quitte à en faire table rase... Est-ce avec l'adieu aux Lumières, la fin du projet dont la Révolution s'était portée héritière?

\section{Peace and love : les bretelles de Sarastro}

Sorti en France à Noël 2006, après un lancement assez tapageur dans le monde anglo-saxon (pour un succès que mon expérience limitée de spectateur provincial ne confirme que modestement) le film de l'anglais $\mathrm{K}$. Branagh ambitionne de donner une adaptation populaire et modernisée de la Flûte Enchantéc. Dans un documentaire télévisé passé sur la chaine 5 le 28 décembre à $20 \mathrm{~h} 45$ le réalisateur nous a conviés non seulement au lancement de son œuvre à Venise, mais à un reportage sur les conditions du 
tournage, avec ses prouesses techniques, accompagné d'interviews des acteurs et de déclarations personnelles qui éclairent (sans trop de fioritures) sur ses intentions, et sa conception d'ensemble. Tels détails ne sont pas superflus s'agissant d'une transposition dont les partis-pris peuvent étonner plus encore que les jongleries psychédéliques auxquelles nous sommes désormais habitués (Vous avez vu "Star War" et «Le Seigneur des Anneaux " demande Branagh aux trois jeunes acteurs dans le rôle des enfants... c'est pareil). Récusant d'entrée toute piété déplacée à l'égard de la littéralité de l'héritage (sinon à l'égard de la musique), le réalisateur dit s'être imposé une "énorme exigence d'imagination " pour faire passer l'émotion, en prenant des libertés d'autant plus licites que Mozart luimême écrivant, dit-il, pour un théâtre populaire a livré un scénario « qui ne tient pas debout ". Il en respectera la trame événementielle comme il transmet la musique avec un réel succès associant la prestation en faux direct et en play-back d'authentiques chanteurs d'opéra. Mais la nouveauté - et la surprise - se dévoilent dans le contenu, la mise en scène, et la signification donnée à l'original mozartien.

On en juge d'entréc : nous avons beau être désormais habitués, ou résignés, au parti-pris de transposition dans une période contemporaine, aux fins de modernisation, le choix d'inscrire la Flûte Enchantée dans le contexte de la Première Guerre mondiale, en 1915 peut-être, comme un épisode qui prend naissance dans les tranchées reconstituées avec quelque fantaisie au coeur d'un paysage de désolation ravagé par la guerre, se veut une provocation hardie. Un général à barbe blanche donne le coup d'envoi à un orchestre militaire de violons qui signent assez cocassement le propos d'instrumentaliser (c'est le cas de le dire) la musique de Mozart.

D'entrée, après un ballet acrobatique et gratuit d'avions de la Première (ou Deuxième) Guerre mondiale, le cadre qui va être celui de la majeure partie du premier acte prend l'allure d'un champ de massacre nocturne, glauque, traversé d'éclairs et jonché d'incendies, où la charge à la baïonnette de soldats dérisoires s'enlise dans le sang et la boue. En back ground s'esquissent fugitivement d'incertaines fraternisations entre soldats. Les bleus britanniques se battent contre les rouges si l'on en croit leur casque - mais en face la forme est identique et les camps sont indéfinis volontairement. C'est « la " guerre qui est évoquée, à la fois historiquement datée mais en même temps dans un flou d'une horreur proche et lointaine. Et de suite on s'interroge sur la finalité de cette mise en scène destinée à justifier la frayeur initiale du héros Tamino : le monstre qui le poursuit dans l'opéra, l'inoffensif serpent de la scène traditionnelle prend les dimensions d'une apocalypse guerrière, chronologiquement proche de nous, sans toutefois s'identifier à celles d'hier, entendons aux affronte- 
ments dont la blessure nous est encore la plus vive, après 1938. C'est une mémoire à demi refroidie qui est invoquée dirait-on, par souci de garder un caractère de généralité à l'interpellation sur son horreur. D'autres cadres vont relayer ce choc visuel initial : ils seront évoqués au fil de leur apparition, à mesure que les acteurs du drame prennent leur place.

Voici Tamino : le prince errant de la fantaisie de Schikaneder est devenu un jeune officier dans le camp des bleus. Du clin d'œil dérisoire de l'orchestre de fantaisie, à la charge à la baïonnette dans laquelle il a entraîné ses hommes, on le découvre beau jeune homme bien anglais, attaché à son devoir patriotique sans trop savoir où il l'entraîne, et pour l'heure blessé, choqué du moins, gisant dans la boue et la nuit. Quand il émerge ahuri, c'est pour être confronté à l'apparition des trois suivantes de la Reine de la Nuit. Éblouissante tache de blancheur, éclair dans la nuit glauque, les voici vues de dos puis de face, avec leurs tenues blanches d'infirmières dotées d'impressionnantes cornettes, celles dont les anglo-saxons aiment à coiffer les bonnes sœurs papistes, mais très exagérées. On se remémore alors les nonnes rouges de la mise en scène québécoise de 1995, mais ici on ne voit pas leurs porte-jarretelles. Toutefois ces anges de la mort, ou plutôt de la vie, n'échappent pas au tempérament qui semble désormais attribué avec une complaisance croissante à ces messagères : lubriques, elles ont de gros nichons et ne résistent pas à la tentation (inaboutie) de se dépoitrailler un instant, avant de palper avidement le héros meurtri en profitant de son inconscience. On va les retrouver au fil de l'opéra sous l'uniforme «britannique » qui ne les flatte pas, renvoyées au monde chtonien des tranchées puis des souterrains, dans leur rôle de séides et messagères de la Reine de la Nuit.

On attend celle-ci, suivant toutes les traditions, et pas simplement pour le rendez-vous vocal de son premier grand air : quel rôle lui sera dévolu ? Le scénariste-réalisateur n'a eu pour elle aucune complaisance. Venue de l'espace (dans une fantaisie sidérale), elle apparaît debout sur la plate-forme d'un lourd char d'assaut, comme la reine du champ de bataille, celle qui commande le mal, intermédiaire entre l'univers chtonien des tranchées et la clarté lunaire dont elle émerge. Sanglée dans un ensemble noir d'une austère coquetterie, elle énonce son message de haine... Mais que représente-t-elle ? Le scénariste n'est pas à l'aise avec ce personnage emblématique de l'" Empire du mal », dans son interview il la situe " à la limite du surnaturel », mais retombe lourdement sur ses pieds : c'est « une fille qui a des problèmes » dit-il assez grossièrement, précisant un peu plus tard que c'est « une femme qui souffre d'avoir été rejetée par un homme », commentant lestement que ce sont des aventures de ce genre qu'on rencontre dans les feuilletons. On dira que Mozart et Schikaneder ne lui 
ont pas facilité la tâche : mais la théâtralisation grandiose du cadre guerrier de l'"Empire du Mal » ne laisse pas d'accentuer le caractère assez dérisoire de sa conduite sous l'égide d'une femme qui a mal vécu son divorce. Dans son dispositif inventé, le scénariste actuel peine visiblement à donner du mystère, et une consistance autre que caricaturale aux héros d'une fantaisie si fortement incarnés dans l'héritage mozartien (et si ambigus déjà).

Il n'a pas mieux réussi, me semble-t-il, dans le personnage de Papageno, qu'il nous revient d'introduire ici. C'est difficile de se présenter comme oiseleur des tranchées. La faute ne revient pas à l'acteur, bel homme de type britannique qui ressemble un peu à Tamino, un troufion comme lui, à cela près que l'un est officier et l'autre du rang. Il tient bien son rôle, au poste qui lui est attribué par le livret, mais accentué par le réalisateur : attaché aux choses de la nature, de la bouffe et de la baise. Branagh ne tourne pas autour du pot, qui dans son commentaire l'expédie en une phrase : "I need some sex». La tendance que nous avions cru voir s'esquisser dans les récentes productions, d'une réévaluation ou survalorisation de Papageno au détriment de son double élitiste, Tamino, ne se confirme pas, ce qui ne veut pas dire que le réalisateur ne lui ait pas fait quelques gracieusetés remarquées en forme d'intermèdes propices à des fantaisies "psychédéliques ». Nous aurons ainsi droit à une séquence de rêve pastoral et érotique de Papageno au second acte, où il se voit entouré d'une troupe d'avenantes Papagena parachutées autour de lui, après qu'il se soit engouffré dans une énorme bouche féminine pulpeuse éclose au milieu d'un champ. Et au final lorsqu'il touche au but de ses vœux, les trois enfants aménagent pour lui et pour Papagena un petit cosy corner au milieu d'une grange de foin, en happy end familial. Mais Papageno ne devient pas le rival de Tamino qui reste le héros du voyage initiatique.

Avant de franchir cette étape, il est indispensable de découvrir, comme le fait le héros, Pamina belle jeune fille, émouvante, de belles dents (comme les autres d'ailleurs, les grands airs obligent). Cette héroïne tonique affronte, si on lui applique une lecture du genre de celle que le réalisateur a réservée à sa mère, un parcours romanesque mais classique : enfant d'un couple désuni, enlevée par son père, un coup de foudre, une tentative de viol, puis un grand désespoir d'amour qui manque tourner en suicide, avant des retrouvailles sublimes et mouvementées. Si nous n'étions si avides de l'attendre aux rendez-vous obligés des grands airs mozartiens, nous dirions que dans ce monde de guerre et d'horreur elle remplit honorablement son contrat. On devine bien qu'elle est faite pour les bals hollywoodiens dont le réalisateur intercale l'image esquissée avec un clin d'œil dans des séquences de rêve qu'il présente en noir et blanc, à la fois pour accentuer le procédé et évoquer les classiques américains. 
Il a fallu d'abord affronter l'épisode aujourd'hui difficile à gérer par les adaptateurs de la capture par Monostatos et ses sbires, qui introduit de manière inélégante à l'entrée dans l'Empire des Lumières, en même temps qu'il confronte à l'obstacle d'un Mozart raciste, qui ne mâche pas ses mots sur les races inférieures et leurs pulsions. À cela heureusement il existe des réponses appropriées, Monostatos n'est plus, évidemment esclave du maître, mais un super vigile, petit chef dans la garde rapprochée - en uniforme - du boss, il ne sera plus condamné à être fouetté mais dégradé avec mesure de son grade de caporal. Pas très noir, juste un peu bronzé pour les besoins du rôle, il est présenté de façon émouvante lui aussi comme porteur de problèmes encore plus complexes que ceux de la Reine de la Nuit : celui de la race, du statut inférieur qui en fait un humilié, du sexe enfin qui le chatouille furieusement, comme Papageno, mais avec visiblement un tempérament de violeur. Fermeté et compréhension de son maître n'y pourront rien, il finira définitivement dans le camp des réprouvés.

Qui sont ses adjoints, uniforme rouge et calot beige. Police politique, Guépéou, SS ou quoi ? À part les hommes de main qui brutalisent mais sans excès, nous allons faire connaissance avec quelques-uns d'entre eux, lorsque Tamino et Papageno introduits dans le sanctuaire vont être soumis à interrogatoire, de part et d'autre d'une table de commissariat un peu miteux. On craint, mais finalement cela ne se passe pas si mal : c'est un «black » qui questionne Tamino, un « jaune » Papageno et ils sont vraiment très bien, plutôt dans le genre " psy " que dans le rôle d'agents de la Stasi. Ils font preuve de compréhension, voire d'une certaine condescendance amusée à l'égard de l'idiot de la bande, Papageno. Et d'une réelle compassion vis-à-vis de Tamino dans l'attente des épreuves qu'il va accepter d'affronter.

Au sortir de l'univers dévasté par la guerre ou plutôt enchâssé en son sein, voici le château de Sarastro, abordé de nuit qui fait pénétrer dans le cadre d'un conte de fées ou fantastique selon que l'on voudra, car ce pourrait être celui de Dracula, révélé de l'extérieur dans un style néogothique à la Viollet-le-Duc ou à la Walt Disney, c'est tout comme. Vu des toits, il est tout encombré de tronçons d'architecture gothique, dans un désordre inachevé, vu d'en bas ses poternes médiévales sont surmontées d'inscriptions morales où se retrouve, mais pour une fois seulement une réminiscence maçonnique. Les espaces intérieurs, sans souci d'unité de style, révèlent les volumes de grands halls et d'amples escaliers néoclassiques, comme dans les films dirait-on, mais initialement encombrés d'échafaudages entre lesquels on se faufile : un monde en construction plutôt encombré, un désordre où évolue une population étrange. 
Le château est-il un havre de paix dans un monde de guerre ? On nous fera visiter au hasard de l'opéra les tentes d'un hôpital militaire dans ses dépendances, accueillantes aux victimes des combats : car Sarastro fait la paix, pas la guerre. Certes il est le chef d'un parti - les rouges qui sont l'équivalent des bleus à la couleur près mais sous le même casque - et luimême revêtira l'uniforme, chevauchant un cheval blanc, mais ce n'est pas sous cet aspect que nous le découvrons initialement, au milieu de son peuple.

Qui sont-ils ? On a déjà vu les cadres du parti. Le petit peuple qui grouille est un peu fantomatique. Gens du peuple, femmes, hommes et enfants, assez pauvrement vêtus, humbles parfois, mais plus que dociles, convaincus, un camp de réfugiés? Des Kosovars, des Albanais, des SDF un peu grunge, avec un souci marqué de pluri-ethnisme accueillant ? Puis on voit cette foule indistincte s'activer, se livrer aux petits métiers de l'échoppe et de la boutique, à des ateliers modestes, ou à l'aménagement du site. Ils ne sont pas malheureux, aptes à faire la fête, célébrer les épisodes par des danses, des trémoussements collectifs de type assez charismatique.

Où sommes-nous ? En Corée du Nord, non sans doute, à Cuba peutêtre. Le culte du chef est présent, Isis et Osiris ne se montrent pas, et la seule allusion au culte du Soleil se glisse sans s'imposer quand une rosace s'illumine un instant. Au moment de la plus forte tension, on priera ici comme dans les tranchées (ou aux États-Unis) pour que les héros qui sont à l'épreuve sortent victorieux. Et Sarastro lui-même - mais quel est-il ? Comme chez Mozart (!) c'est lui en dernier recours qui doit délivrer sinon le secret, le message : mais cela demande, de la part de ses protégés comme de nous-mêmes un acte de confiance qui ne va pas de soi.

Finalement, je sais gré au réalisateur, sur fond de mes expériences anciennes ou récentes en flûtologie de n'avoir pas transformé Sarastro en Staline. Malgré le registre de sa voix, et le rôle qu'il assume, c'est un jeune père bien sympathique. On ne l'a pas voulu distant au premier contact, avec son nez retroussé, sa bonne bouille, et surtout ses bretelles qui signent une forme de sans façon bien anglo-saxonne. Ce n'est qu'ensuite qu'on le verra, en général des forces de la paix, jamais en pontife. Il garde l'humanité d'un père dont le mariage a mal tourné avec une virago qu'il verra avec compassion s'écraser avec ses complices dans la scène finale de l'ascension du mur du château par les Dalton, pour se perdre dans l'obscurité. Il n'existe pas de réconciliation possible avec les forces du mal incarné, non autrement défini sinon - ce qui n'est pas rien - que la nuit cautionne de son voile l'univers glauque de la guerre et du massacre tel qu'on l'a découvert au début. 
Sarastro incarne les forces du bien : ce qui n'est pas évident d'entrée, prévenus que nous sommes en sa défaveur par l'épisode de l'enlèvement de sa fille avec ses dégâts collatéraux (tentative de viol, bavures), ses auxiliaires revêches et l'abord du château de Barbe Bleue peu attractif. Que ce soit par les poternes médiévales ou les toitures transformées en bordel kitsch néogothique, l'accès de la forteresse est bien gardé. Puis Sarastro, malgré tout ce que j'ai dit de sa bonne bouille, ne transige pas sur l'article : pas question de capituler devant le camp du mal, et surtout même si nous éviterons de parler goulag ou Guantanamo, un lavage de cerveau s'impose sans discussion, il est gradué, adapté suivant le sexe et le statut social et culturel. Et l'on ne peut échapper puisque c'est dans le livret, au couplet infâme sur la débilité, pire, la perversité féminine, qui justifie toutes les âneries que l'on pourra écrire sur les limites de Mozart et des Lumières associées.

Sous le pouvoir de Sarastro quel brave jeune officier que Tamino depuis le début, il va où est son devoir, au casse-pipe, pour la patrie, à l'hôpital. À la recherche de la bien-aimée par qui s'opère le miracle de la haine et de la fureur initialement tournées contre Sarastro jusqu'à l'initiation acceptée chez peace and love. Finalement, il persévère dans son profil bon élève réceptif à l'explication de Sarastro et au lavage de cerveau soft par les deux apparatchiks. Fait-il cela par amour ou par conviction nous demandent les exégètes depuis longtemps ? Les deux mon général et le problème n'est pas approfondi plus qu'il ne convient, pas plus que dans Mozart assez élusif sur ce point. C'est apparemment sans états d'âme qu'il a revêtu l'uniforme de l'armée rouge (je plaisante) et c'est normal puisque ce sont les combattants de la paix. Tamino réussit aux examens dans la catégorie supérieure.

Peut-être injuste envers son impassibilité anglo-saxonne, je me penche avec compassion sur le sort de Pamina : car c'est elle qui dans cette mise en scène subit les épreuves les plus rudes surajoutées parfois par le réalisateur sur le canevas mozartien ; échappant pour la seconde fois à la lubricité de Monostatos elle a avec sa mère une dernière entrevue assez " hard ». La méchante femme dans un épisode fantasmatique va jusqu'à la ligoter (la crucifier presque) aux ailes d'un moulin - invention gratuite dont elle incendie les extrémités. Ralliée par son père à la bonne cause, Pamina est prête à devenir à parité avec son amoureux l'héroïne des épreuves initiatiques dont la mise en scène, abusant des licences que permet le cinéma par rapport au théâtre a fait un épisode grandguignolesque, en particulier dans l'épreuve de l'eau, digne d'un film américain : c'est dans un rapide à mi-chemin entre jacuzzi et tsunami que barbotent les deux élus. 
En play-back la voix off des deux héros parvient à préserver l'instant magique de ce haut sommet de l'incantation mozartienne. Et Pamina n'a même pas perdu son top.

Quant à la suite vous la connaissez : un brevet professionnel et un livret de famille pour Papageno invité à copuler et à jouir sans contrainte, un brillant avenir pour le couple d'élite qui a triomphé des obstacles. La Reine de la Nuit dans un dernier râle de fureur, a pu entrapercevoir de l'embrasure de la fenêtre à laquelle elle s'accroche, la surprise-party, verres de champagne en main qui scelle l'accomplissement de l'aventure d'amour et de passion.

Mais nous ne sommes pas au bout du compte : comme dans tous les films (j'allais dire américains) l'aventure des héros s'inscrit dans une apothéose finale.

Voici le triomphe du soleil dont les rayons font naitre une nature verdoyante de campagne anglaise sur le champ de bataille dévasté des combats nocturnes. Lunivers enténébré et chtonien qui a dominé la majeure partie du film s'éclipse, livrant le fin mot de l'énigme : la lutte des Lumières contre la Nuit, c'est celle du bien contre le mal, certes, mais essentiellement de la paix contre la guerre.

Peace... and Love, car l'amour sous ses différentes expressions - au ras des pulsions comme dans son expression la plus épurée - voit son triomphe dans cette apothéose finale. Mais ne nous y trompons pas c'est une passion corrigée, guidée d'une main ferme par ceux qui savent, qui permet à l'issue d'une initiation héroïque d'accéder à la sagesse.

Ceux qui savent? Qui sont finalement les habitants de la forteresse gothico-kitsch de Sarastro dans le scénario actuel ? Une secte? Des pauvres bougres, réfugiés ou marginaux ? Une milice, éventuellement musclée ? Une élite ? Le flou se révèle dans le parterre final des hommes et des femmes de bonne volonté qui saluent le soleil : vous et moi. Mais Sarastro lui-même disculpé de la cruauté initiale dont il était crédité, apparait comme un chef respecté, aimé, scrupuleux mais inflexible sur le but à atteindre : en somme le « prince bienveillant "... en chemise et bretelles, comme en uniforme.

Rêve d'enfants ou rêve d'adultes ? À qui s'en remettre du projet «peace and love »? Chez Mozart nous n'avons pas attendu Régis Debray pour discerner en Sarastro les traits du monarque éclairé des Lumières. Et derrière la transparente fiction maçonnique, le groupe fermé des élus se devinait plus aisément que la troupe imprécise du film enrichie de composantes ambiguës par des stratifications du $X X^{c}$ siècle. Paternaliste plus que totalitaire, l'utopie de Sarastro brode sur le rêve plus encore que de l'avè- 
nement des Lumières, de la paix universelle. Ni Staline, ni Bush car l'axe du bien récuse la guerre et ses cruautés, cette fable de Noël est enduite de bons sentiments qui n'ont rien de subversif - les Lumières a minima.

Loriginalité - mais qui témoigne à sa manière de l'air du temps - est d'avoir transposé cette leçon dans un cadre " semi contemporain " plutôt qu'actuel. Pourquoi avoir choisi les tranchées, 1915 plutôt que les conflits d'aujourd'hui ? Prudence élémentaire nous l'avons dit. Ruse dont il restera à apprécier si elle est opératoire ou non, et si la mémoire amortie de ce que mes étudiants de 1968 appelaient "la grande boucherie impérialiste " est adaptée à revivifier ce qu'on aurait appelé, hier également, une prise de conscience, que l'influx lointain (oh combien tamisé !) des Lumières du XVIII' siècle n'est plus susceptible de susciter.

D'une réalisation qui cherche la provocation destinée à faire passer dans le grand public " une musique pour vieux " (interview d'un des ouvriers du studio de tournage), on retiendra comme l'a fait la presse les prouesses de la mise en scène, de l'invention du cadre, des gadgets " psychédéliques " gratuits. Et aussi le succès discutable dans la difficile coordination entre le jeu, le chant des acteurs, le cadre imposé, la fidélité et la trahison à l'opéra de Mozart.

Mais au-delà de ces prouesses, quelle est la portée du message ? Avec simplicité K. Branagh déclare avoir voulu aborder « la philosophie et le mystère de la vie " avec pour but de démontrer que "l'amour et le bonheur sont possibles ». Louables objectifs, qui tolèrent avec la fiction de Schikaneder et Mozart les plus grandes libertés : les hôtes du château ne sont pas des francs-maçons, « ils travaillent pour la paix ». Ramenée aux dimensions d'une organisation non gouvernementale, cette association aux limites d'une secte, n'est pas sans ambition à travers la personne de son chef : Sarastro annonce " Gandhi, Martin Luther King et Jésus Christ à la fois »... Holà ! Prudence... Dans sa naïve démesure cette ambition porte-telle témoignage, au crépuscule des Lumières, de la formulation élémentaire «peace and love » de notre monde assiégé par la guerre ? Mais aussi de la grande plasticité du génie mozartien apte à résister aux traitements les plus rudes?

Et finalement une question pour départager... Qui est donc Sarastro avec ses bretelles?

\section{La fin des Lumières ?}

Trois variations sur "La Flûte " dans ces dix dernières années. On dira le choix hétérogène d'Aix à Moscou, de l'opéra au film qui se veut 
people : il demeure significatif de l'image des Lumières aujourd'hui à travers les options proposées.

Dans leur parti pris de modernisation, elles ont jeté par-dessus bord, sinon par référence plus ou moins ironique les oripeaux maçonniques quitte à les remplacer par un sacré de pacotille (Carsen 1995), ou en assumant la responsabilité d'une transposition anachronique dans un aujourd'hui décalé (Branagh 2006), ou encore chaud (Moscou 2006). Toutes, de façons différentes, prennent leurs distances par une dose d'ironie plus ou moins appuyée parfois grinçante voire massacrante ; l'érotisation est un ingrédient apprécié, en contrepoint avec une dramatisation qui fait prévaloir le contraint, le tragique ou le glauque dans deux cas au moins sur trois. Le sens du sacré se perd dans la dérision ou dans l'enflure, les héros fatiguent, seul Papageno se porte bien on s'en réjouit. Mais au bilan, les Lumières se négocient au rabais, voire s'abîment dans une apocalypse finale.

Les deux versions « anglo-saxonnes » ont en commun un happy end, porteur d'un message optimiste que j'ai caricaturé, mais à peine, à l'enseigne du " tout le monde il est beau... " ou la réconciliation générale dans la version de 1995, de peace and love dans celle du film en 2006. Mais c'est à l'issue d'un cheminement différent : doit-on en conclure qu'en dix ans au tournant de l'an 2000 le tableau s'est assombri, l'oecuménisme soft qui nie la réalité du combat entre ombre et lumière, emblématisé par le conflit homme femme, étriqué aux dimensions d'un malentendu provisoire, faisant place en 2006 à l'affirmation qui se veut tragique de la réalité du mal, désigné comme la guerre, sans pour autant nous permettre d'en discerner avec vraisemblance la source (la Reine de la Nuit ?) ni le remède (le sauveur?), renvoyant à la conversion miraculeuse des esprits pour faire triompher la paix et l'amour?

Le mérite de la version moscovite est bien celui de la provocation. L'univers des Lumières est celui du totalitarisme, il a son chef et c'est Staline, l'initiation est un endoctrinement, la cité utopique une prison, même si l'aspect « goulag » s'atténue par la dérision plus que par l'excès. Mais s'il n'y a pas d'équivoque sur l'identification de Sarastro-Staline, le trouble demeure s'agissant de la Reine de la Nuit. Force nocive certes mais que représente-t-elle au fond? Il faut attendre l'ultime séquence, où l'on voit apparaitre avions et missile du 11 septembre 2001 pour avoir une piste : la nuit c'est la Terreur, - l'axe du mal ? - mais c'est lui qui l'emporte finalement dans cette lecture nihiliste où les fléaux s'autodétruisent.

Leffet boomerang de l'effondrement des régimes totalitaires et du collapsus des révolutions du $\mathrm{XX}^{\mathrm{e}}$ siècle qui se réclamaient des Lumières frappe en retour avec une cruauté caricaturale l'univers enchanté de 
Mozart. Limpact dévastateur est tel que finalement rien ne lui résiste : Staline est mort, mais finalement Bush grimace vilainement sous les coups du commando suicide des kamikazes féminins.

Les dernières décennies ont vu prévaloir, sur des arguments forts et qui se veulent définitifs, l'idée que la Révolution est terminée : la réaction en chaîne qui s'amorce aujourd'hui s'en prend aux Lumières que la tradition progressiste (on aurait dit hier la vulgate) lui associait comme un accomplissement. Les Lumières et les philosophes aux origines de la grande subversion : dans sa version positive comme dénonciatrice le cliché a fait son temps. La tendance lourde aujourd'hui est au désamarrage de deux esquifs qui prennent l'eau : et le pamphlet de Régis Debray Aveuglantes Lumières ${ }^{18}$ très explicite sur ce point, dénonce brutalement la « conjonction en bois " de la formule "La Révolution et les Lumières ", «ce et mat et machinal ${ }^{19}$ qui les associe traditionnellement... Une sorte de baiser qui tue. Voilà une vieille chanson remise au goût du jour. Mais la nouveauté est sans doute que de l'opération les Lumières ne sortent pas indemnes comme certains l'espéraient. C'est non pas pour le message qu'ils ont transmis, mais pour ce qu'ils ont méconnu de l'éruption à venir que Voltaire et consorts, philosophes de boudoir, sont pris à partic.

Et Mozart dans tout cela ? Nous ne l'avons pas perdu de vue. Mais pour Debray le « chérubin des Lumières qui nous fait miroiter l'heure exquise " ${ }^{20}$ n'échappe pas à l'anathème : "Il n'a rien deviné de l'âge des foules ni des noirs tressaillements de 93-94 $»^{21}$. Pour un peu on lui enverrait le commando des amazones de la Reine de la Nuit. La-t-il mérité ?

Lessayiste d'aujourd'hui revenu non seulement des errements révolutionnaires qu'il s'agisse dit-il du " dégagement de chaleur des journées révolutionnaires, les rots et les pets du gros animal $\aleph^{22}$, ou de sa mise en système «le guindé, le blafard, le cireux, disons le Comité de Salut Public »23, mais aussi de l'illusion du progrès et de la propagation des rayons de la raison, se voit réduit nous confie-t-il à promener "l'idée ingrate " d'une "gauche tragique " ${ }^{24}$ qu'il prêche dans le désert.

Sans doute ne perçoit-il plus les sons de ce « concert de flûte dans le lointain » qui, de la traversée des épreuves pour Pamina et Tamino, celle de la mort assumée et dominée, à cette grande initiation collective aux valeurs nouvelles d'émancipation humaine transmises par les philosophes des Lumières qu'a été la Révolution française, assure la continuité de ces deux séquences indissociables.

(18) Régis DEBRaY, Aveuglantes Lumières, op. cit., note 1.

(19) Jbid., p. 107.

(20) /hid., p. 144.

(21) Ibid., p. 144

(22) lbid., p. 144

(23) Ibid., p. 110.

(24) Ibid., p. 179. 
La Révolution, par la voix de Robespierre et quelques autres, a dénoncé les philosophes qui vivaient dans l'ombre des grands, mais SaintJust a écrit « le XVIII siècle doit être mis au Panthéon ", et Mirabeau avait reconnu « nous avons des avances d'idées ».

Pour comprendre ces appréciations contradictoires, n'oublions pas la dualité des Lumières, ce contraste entre ombre et clarté qui est précisément au cour de l'opéra mozartien, et qui s'accentue au fil du siècle. Pour illustrer la couverture des deux tomes qui ont été consacrés voici dix ans au siècle des Lumières sous ma direction ${ }^{25}$, j'avais choisi deux images symboles : "Glad Day " de William Blake (1780) où l'homme des Lumières se montre, nu, jubilatoire sur fond d'un soleil rayonnant, mais aussi le frontispice que donne alors Goya à sa série des Caprices : le dormeur qui s'abandonne, la tête couchée entre ses bras sur une table est assiégé dans son rêve par le vol des oiseaux de nuit. Et le commentaire dit : « le sommeil de la raison enfante des monstres ". Sage mise en garde, avant de se laisser aller à en faire le procès. Elle éviterait de se retrouver dans la posture du personnage que j'ai rencontré en feuilletant, à la recherche des Lumières, dans mes volumes d'Images et Récits et que le caricaturiste invective irrespectueusement : «Au milieu de l'éclat le plus pur, tu restes dans le clair obscur $»^{2 \hbar}$.

Est-il convenable de s'arrêter ainsi sur une pirouette ? On me suggère que non. Contaminé par Mozart, j'ai à la hauteur de mon génie propre qui est modeste, entremêlé le sérieux et le comique. Mais le sérieux l'emporte dans ce souci de renifler l'air du temps. Cette esquisse impressionniste mériterait peut-être de susciter des prolongements. Quelle jolie thèse, si cela n'a déjà été fait que de remonter le temps en amont des années 1960 jusqu'à la création de l'opéra en 1791. Moyen de suivre la façon dont le Singspiel mozartien s'est différencié des féeries à l'italienne dans le style de Gozzi (voire d'héritages beaucoup plus anciens encore), pour se charger du message idéologique qui en a fait une illustration emblématique de l'esprit des Lumières ${ }^{27}$.

(25) Le Siècle des Lumières - L'apogée, 1750-89, 2 vol,, 1210 p., Coll. Peuples et Civilisations, M. Vovelle, G. Lemakchand, M. Gi,Li, M. Cubells, sous la dir. de M. Vovelle, Paris, PUF, 1997. Alors que la périnde revenait au programme de l'agrégation, l'éditeur a jugć opportun en 2005 de pilonner cet ouvrage trop cher pour le candidat moyen.

(26) Michel Voveli.F, L a Révolution française. Images et récit, op. cit., tome Il, p. 265, note 5.

(27) Mais pourquoi s'en tenir à une vision rétrospective? Au risque d'apparaître définitivement comme un cinéphile amateur de vocation tardive, je conseille le dessin animé japonais pour grand public qui sort sous le titre de "Contes de Terremer ". Il y a là une Reine de la Nuit fort méchante dans son châtcau, un sorcier-mage détenteur de la sagesse qui l'affronte mais en solitaire et deux enfants garçon et fille auxquels est transmis le grand secret que l'immortalité est un leurre, et la mort la fin naturelle de la vie. Esquisse élémentaire de dialogue Orient-Occident, fonds commun des contes el légendes... Qu'on ne s'y trompe pas, cette production un peu prétentieuse et sans grande portée, n'est que la transcription pour adultes mais avec des moyens identiques (l'extrême violence des images) du quotidien dont se nourrissent les enfants sur les écrans de télévision. 
Il va de soi que ce support musical ponctuel ne représente qu'une voie d'accès, séduisante peut-être mais limitće à l'étude de l'image héritée des Lumières dans son évolution depuis deux siècles, en passant par la Révolution... Sur ce point je renvoie sans en partager vraiment l'esprit à l'essai incisif de Jean-Marie Goulemot, cité en introduction : Adieu les philosophes, que reste-t-il des Lumières ?, soldes avant inventaire, vide grenier qui d'une certaine façon justifie et illustre les métamorphoses que nous avons rencontrées dans les présentations actuelles de l'opéra de Mozart. Compromises pour avoir précédé la Révolution - parce qu'elles l'ont préparée pour les uns, parce qu'elles n'ont pas su l'anticiper pour les autres -, voici les Lumières ballottées au gré des vicissitudes de notre imaginaire contemporain, entre ses espoirs et ses peurs, dans un monde où les maître mots à la mode sont ceux d'illusion et de désenchantement.

Michel VovelLE

Professeur émérite Université de Paris I Panthéon-Sorbonne 17 rue de la Sorbonne 75231 Paris cedex 05 